

\title{
II. Cas d'un alliage métallique liquide imprégnant une structure poreuse
}

J. Gillot, P. Stecher, B. Lux

\section{To cite this version:}

J. Gillot, P. Stecher, B. Lux. II. Cas d'un alliage métallique liquide imprégnant une structure poreuse. Revue de Physique Appliquée, 1970, 5 (3), pp.565-572. 10.1051/rphysap:0197000503056500 . jpa00243429

\section{HAL Id: jpa-00243429 https://hal.science/jpa-00243429}

Submitted on 1 Jan 1970

HAL is a multi-disciplinary open access archive for the deposit and dissemination of scientific research documents, whether they are published or not. The documents may come from teaching and research institutions in France or abroad, or from public or private research centers.
L'archive ouverte pluridisciplinaire HAL, est destinée au dépôt et à la diffusion de documents scientifiques de niveau recherche, publiés ou non, émanant des établissements d'enseignement et de recherche français ou étrangers, des laboratoires publics ou privés. 


\title{
II. CAS D'UN ALLIAGE MÉTALLIQUE LIQUIDE IMPRÉGNANT UNE STRUCTURE POREUSE
}

\author{
par J. GILLOT, P. STECHER, B. LUX \\ Institut Battelle, Genève, Suisse
}

\begin{abstract}
The mechanical stability of a liquid metallic ailoy that impregnates the porous superficial layer of a coating that is submitted to an acceleration depends on (a) the shape of the porosity, which should preferably be fine and with a low degree of interconnexion (b) the wettability of the solid by the liquid, which should be good on the internal surface of the porous layer, and poor on its external surface (c) the presence of superficial oxide layers that obturate the openings of the pores. In the case of a coating deposited on a gas turbine blade, the stability of the liquid requires a very fine porosity with a low interconnexion degree. In the case of a coating deposited on a static part, a fine porosity will retain the liquid, whatever its degree of interconnexion.
\end{abstract}

1. Introduction. - Les revêtements de protection des métaux contre l'oxydation sont, en général, des couches solides compactes. Cependant, l'emploi de telles couches ne permet pas toujours d'obtenir les caractéristiques voulues. L'adjonction d'une phase liquide peut parfois résoudre ce problème, grâce à la mobilité ainsi conférée à certains constituants du revêtement. Ces constituants peuvent alors assumer des fonctions telles que le remplissage et la guérison des fissures. Néanmoins, leur mobilité fait apparaître un nouveau problème : celui de leur rétention dans les diverses conditions d'utilisation de la pièce recouverte.

C'est ce problème de la stabilité mécanique d'une phase liquide comprise dans un revêtement composite qui va être étudié ici, dans le cas particulier d'un revêtement de protection du niobium contre l'oxydation qui est constitué d'une couche de solide poreux à porosité ouverte imprégnée d'un alliage métallique liquide à faible viscosité. Le cas d'un revêtement constitué d'une couche d'un verre visqueux déposé à la surface d'un solide compact a été étudié dans la première partie du présent mémoire. La réalisation, les caractéristiques physico-chimiques et les performances de ces revêtements ont été décrites par ailleurs $[1,2,3]$.

Parmi les diverses sollicitations auxquelles peuvent être soumis les revêtements de protection du niobium, seules seront considérées ici les accélérations qui peuvent être dues à la pesanteur, à une force centrifuge ou à des vibrations. Le liquide, que les accélérations tendent à éjecter de la couche poreuse, y sera maintenu principalement par les forces de capillarité dont l'action dépend du type de porosité de la couche, de la rugosité de sa surface et de sa mouillabilité par le liquide.

2. Influence de la forme de la porosité sur la stabilité du liquide. -2.1 LES DIVERS TYPES DE POROSITÉ POSSIBLES DANS UN REVÊTEMENT. - Les pores présents dans le revêtement solide peuvent être classés en trois catégories (Fig. 1) :

- les pores fermés,

- les pores simples qui sont assimilables à un tube dont une extrémité a la forme d'un cul-de-sac et dont l'autre extrémité débouche à la surface libre de la

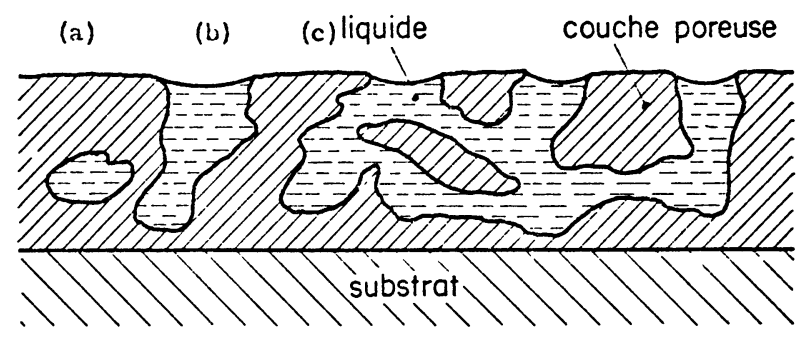

FIG. 1. - Couche poreuse avec des pores
a) Fermé
b) Simple
c) Complexe

couche poreuse. On peut représenter schématiquement ces pores par des cylindres inclinés par rapport à la surface de la couche poreuse (Fig. 2),



Fig. 2. - Représentation schématique d'un pore simple.

- les pores complexes, qui forment une porosité ouverte et interconnectée, qui débouche à la surface libre de la couche poreuse par plusieurs ouvertures (Fig. 1c). On peut assimiler schématiquement un pore 
complexe à un réseau de pores cylindriques interconnectés de diamètres différents débouchant en plusieurs endroits à la surface libre de la couche poreuse (Fig. 3).

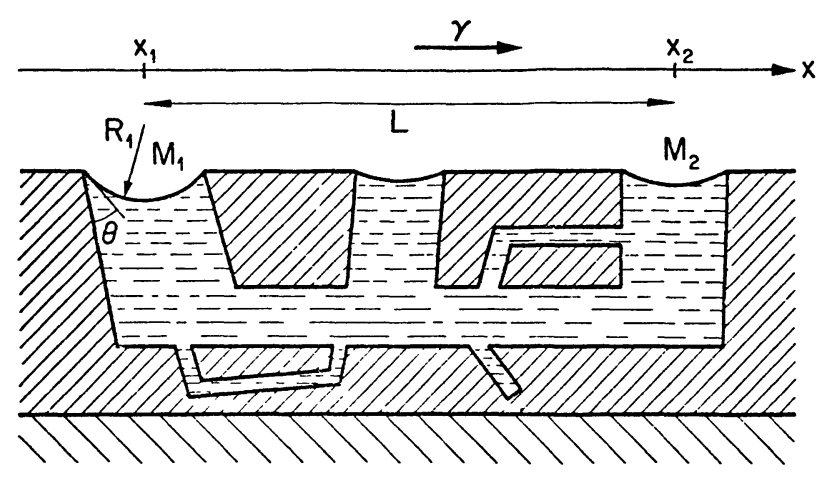

Fig. 3. - Représentation schématique d'un pore complexe.

2.2 STABILITÉ DU LIQUIDE DANS UN PORE COMPLEXE EN L'ABSENCE DE PELLICUle D'OXYDE A LA SURFACE DU LIQUIDE. - Considérons le pore de forme complexe représenté schématiquement dans la figure 3 et supposons qu'il soit rempli de liquide et que les surfaces libres dont l'éloignement parallèlement à l'accélération $\gamma$ est le plus grand, soient les ménisques $M_{1}$ et $M_{2}$ d'abscisses $x_{1}$ et $x_{2}$.

Nous supposerons également que pour $x>x_{2}$, le liquide mouille mal la surface extérieure de la couche solide poreuse. L'accélération impose dans le liquide de densité $\rho$ un gradient de pression $\mathrm{d} P / \mathrm{d} x=\rho \gamma$.

Si les pores sont suffisamment fins, les ménisques ont une forme sphérique. Les pressions $P_{1}$ et $P_{2}$ dans le liquide derrière les ménisques $M_{1}$ et $M_{2}$ sont données par les relations:

$$
P_{0}-P_{1}=\frac{2 \sigma}{R_{1}} \quad \text { et } \quad P_{0}-P_{2}=\frac{2 \sigma}{R_{2}}
$$

où $P_{0}$ est la pression de l'atmosphère ambiante, $R_{1}$ et $R_{2}$ sont les rayons de courbure des ménisques comptés positivement si le ménisque est concave et négativement si le ménisque est convexe, et où $\sigma$ est la tension superficielle du liquide.

D'autre part,

$$
P_{2}=P_{1}+\rho \gamma\left(x_{2}-x_{1}\right)
$$

d'où

$$
2 \sigma\left(\frac{1}{R_{1}}-\frac{1}{R_{2}}\right)=\rho \gamma\left(x_{2}-x_{1}\right)=\rho \gamma L .
$$

A l'équilibre, le ménisque $M_{1}$ est donc toujours plus concave que le ménisque $M_{2}$.

La valeur maximale de $L$ pour laquelle le système est stable est déterminée par les valeurs extrêmes possibles de $1 / R_{1}$ et $1 / R_{2}$.

La valeur maximale possible de $1 / R_{1}$ correspond au cas où le ménisque est légèrement en retrait par rapport à l'embouchure du pore. Le ménisque sphéri- que se raccordant avec la paroi cylindrique du pore avec l'angle de contact $\theta$ (déterminé par la mouillabilité de la surface intérieure du solide par le liquide), on $\mathrm{a}$ :

$$
R_{1}=\frac{r_{1}}{\cos \sigma}
$$

$r_{1}$ étant le rayon du pore.

La valeur minimale de $1 / R_{2}$ qui correspond à un ménisque stable dans tous les cas est $1 / R_{2}=0$, ce qui correspond à un ménisque plan.

Ceci se produit si $P_{2}=P_{0}$. Le ménisque plan est alors stable car la sortie d'une petite quantité de liquide fait augmenter l'énergie totale du système en augmentant la surface libre du liquide.

Le liquide est donc stable dans tous les cas dans le pore complexe si

$\frac{2 \sigma \cos \theta}{r_{1}}>\rho \gamma L$ soit $\quad r_{1} L<\frac{2 \sigma \cos \theta}{\rho \gamma}=(r L)_{\max }$.

Application à un revêtement situé sur une pièce statique. - Le liquide n'est soumis qu'à l'accélération de la pesanteur.

Dans le cas d'une couche poreuse imprégnée d'un alliage d'étain-aluminium qui la mouille bien, on a

$$
\begin{aligned}
\sigma \cos \theta & \sim 800 \text { dynes } / \mathrm{cm} \\
\rho & \sim 4 \mathrm{~g} / \mathrm{cm}^{3} \\
\gamma & \sim 10^{3} \mathrm{~cm} / \mathrm{s}^{2} \\
(r L)_{\max } & \sim 0,4 \mathrm{~cm}^{2}
\end{aligned}
$$

soit, pour des pores élémentaires d'un diamètre de 10 microns,

$$
L_{\max } \sim 800 \mathrm{~cm}=8 \mathrm{~m} .
$$

Pour une pièce de dimension inférieure à $8 \mathrm{~m}$, le liquide sera stable même si la porosité est entièrement interconnectée.

Application à un revêtement situé sur une aube de turbine à gaz. - A titre d'exemple, on considère un revêtement situé sur une aube de turbine à gaz d'un rayon $R_{t}=40 \mathrm{~cm}$ qui tourne à environ 10000 tours $/ \mathrm{min}$ $(\omega=1000 \mathrm{rd} / \mathrm{s}$. En prenant pour le revêtement les mêmes valeurs de $\rho$ et $\sigma \cos \theta$ que ci-dessus, on obtient $(r L)_{\max } \sim 10^{-5} \mathrm{~cm}^{2}$, soit pour des pores élémentaires d'un diamètre de 10 microns,

$$
L_{\max }=200 \text { microns . }
$$

2.3 Stabilité DU LIQUIDE DANS UN PORE SIMPLE, EN L'ABSENCE DE PELliCUle D'OXYDE A LA SURFACE DU LIQUIDE. - On considère maintenant le cas des pores simples qui débouchent par une seule ouverture à la surface extérieure de la couche poreuse, en faisant, comme précédemment, l'hypothèse que le liquide ne mouille pas la surface extérieure de la couche poreuse.

On va montrer que, si le diamètre du pore est inférieur à une certaine valeur, le liquide est stable 
dans le pore. Il ne sera possible de calculer ici qu'un ordre de grandeur pour cette valeur limite. Cela est dû à la complexité des diverses configurations de pores possibles et au fait que, sauf dans des cas très simples, les surfaces des ménisques ne peuvent pas être représentées par des équations simples.

La figure 4 représente un pore simple cylindrique qui fait avec la surface libre de la couche poreuse un angle $\alpha$. Supposons que ce pore soit en train de se vider : une goutte de liquide située en $G$ est en train de se former et va bientôt se détacher du ménisque.



Fig. 4. - Ejection du liquide d'un pore simple : Formation d'une goutte.

Pour que cela se produise, il faut que la différence de pression hydrostatique entre la goutte $\mathrm{G}$ et le ménisque $\mathbf{M}$, causée par l'accélération $\gamma$, soit supérieure à la différence de pression due aux forces de capillarité et à la courbure des surfaces libres de liquide, soit :

$$
\rho \text { MG. } \gamma>\frac{2 \sigma}{R_{\mathrm{M}}}+\frac{2 \sigma}{R_{\mathrm{G}}}
$$

$R_{\mathrm{M}}$ et $R_{\mathrm{G}}$ étant les rayons de courbure des surfaces du ménisque et de la goutte, supposées approximativement sphériques.

$R_{\mathrm{M}}$ est donné par la relation

$$
R_{\mathrm{M}}(1+\cos \theta)=2 r-a
$$

où $a$ est l'épaisseur du « col » qui sépare la goutte du reste du liquide.

La valeur maximale de MG est de l'ordre de $2 r$. En effet, si la goutte s'allonge trop, elle se sépare du reste du liquide et n'a alors plus d'influence sur la stabilité du reste du liquide.

La condition de stabilité peut alors s'écrire

$$
2 r \rho \gamma<\frac{2 \sigma}{R_{\mathrm{M}}}+\frac{2 \sigma}{R_{\mathrm{G}}}
$$

ce qui est toujours vérifié si

$$
2 r \rho \gamma<\frac{2 \sigma}{R_{\mathrm{M}}}=\frac{2 \sigma(1+\cos \theta)}{2 r-a}
$$

soit

$$
r(2 r-a)<\frac{\sigma(1+\cos \theta)}{\rho \gamma} .
$$

Cette condition est toujours remplie si

$$
r^{2}<\frac{\sigma(1+\cos \theta)}{2 \rho \gamma}=r_{\max }^{2} .
$$

On retrouve ici une valeur très voisine de celle obtenue dans le cas d'un pore complexe. En effet, en appliquant l'expression $(r L)_{\max }$ au cas d'un pore simple avec $L=2 r$, on obtient $r_{\max }^{2}=(\sigma \cos \theta / \rho \gamma)$ qui est bien du même ordre de grandeur que

$$
(\sigma(1+\cos \theta) / 2 \rho \gamma) .
$$

Application à un revêtement situé sur une pièce statique ou sur une aube de turbine à gaz. - En prenant les mêmes valeurs numériques que précédemment, on obtient :

- pour la pièce statique : $r_{\max }^{2} \sim 0,2 \mathrm{~cm}^{2}$ soit $r_{\text {max }} \sim 0,4 \mathrm{~cm}$,

- pour l'aube de turbine : $r_{\max }^{2} \sim 5 \times 10^{-6} \mathrm{~cm}^{2}$ soit $r_{\max } \sim 22$ microns.

3. Influence de la rugosité de la surface extérieure du solide et de sa mouillabilité sur la stabilité de la phase liquide. - Dans les calculs qui précèdent, on a supposé que le paramètre $L$ (voir la Fig. 3), qui est la plus grande distance entre deux points de la surface libre du liquide parallèlement à l'accélération, est en même temps la plus grande distance entre deux embouchures du même pore complexe parallèlement à l'accélération. On va maintenant examiner dans quelles conditions cette égalité est vérifiée et montrer comment la rugosité et la mouillabilité de la surface extérieure du solide conditionnent la stabilité du liquide.

3.1 REPRÉSENTATION SCHÉMATIQUE DE LA RUGOSITÉ. - La surface du solide n'est pas lisse, mais présente une certaine rugosité. On peut représenter schématiquement cette rugosité en donnant aux cylindres qui schématisent les pores des parois qui ne sont pas lisses mais qui présentent des microfissures parallèles à l'axe du cylindre (Fig. 5). De même, on peut représenter la rugosité de la surface extérieure de la couche poreuse par des fissures parallèles à l'accélération.

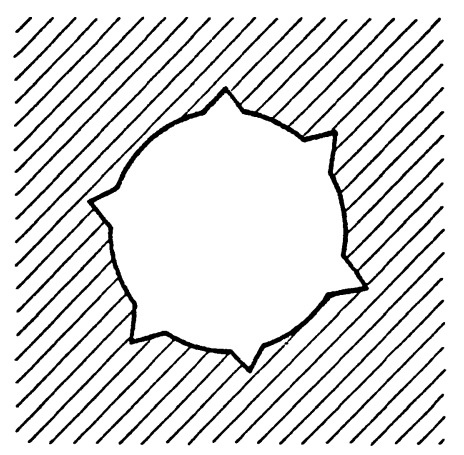

Fig. 5. - Profil d'un pore rugueux. 
Les microfissures sont caractérisées (Fig. 6) par l'angle dièdre d'ouverture $\beta$ et par le rayon de courbure $r_{3}$ du fond de la fissure qui est assimilé à une surface cylindrique. Dans une fissure partiellement remplie de liquide, au point d'abscisse $x_{4}$, le ménisque, qui peut être convexe ou concave, a une forme localement assimilable à un cylindre de rayon $r_{4}$.



Fig. 6. - Coupe d'une microfissure partiellement remplie de liquide.

L'influence de la rugosité de la surface du solide et de sa mouillabilité sur la stabilité de la phase liquide peut être déduite de l'étude du comportement du liquide qui se trouve dans une telle fissure.

3.2 COMPORTEMENT D'UN LIQUIDE QUI MOUILLE MAL LA SURFACE EXTÉRIEURE DE LA COUCHE POREUSE. Le cas considéré ici correspond à celui d'un revêtement de protection contre l'oxydation placé dans des conditions normales de fonctionnement. Au contact avec le gaz oxydant, la couche poreuse est recouverte d'une pellicule d'oxyde que le liquide mouille mal.

Dans ce cas, le ménisque au-dessus d'une masse de liquide située dans une fissure est convexe (Fig. 7), car on a

$$
\theta+\frac{\beta}{2}>\frac{\pi}{2}
$$

a)
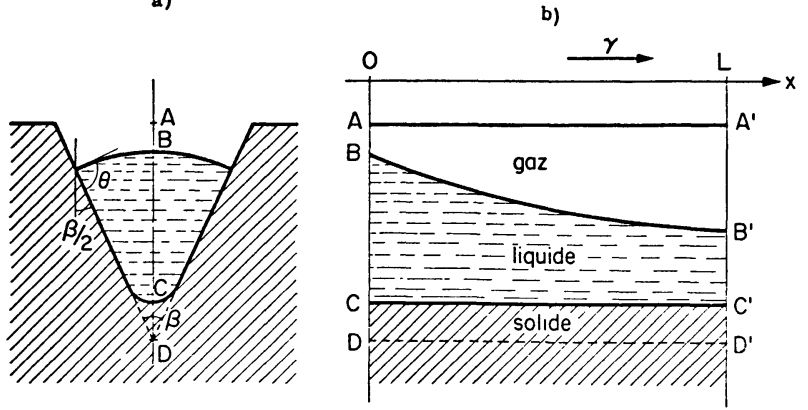

Fig. 7. - Microfissure partiellement remplie d'un liquide qui en mouille mal les parois.

a) Coupe transversale.

b) Coupe longitudinale selon $\mathrm{AD}$ du profil à l'équilibre.
Si la fissure est reliée par le liquide au pore complexe considéré au paragraphe 2.2 et pour lequel $P=P_{0}$ en $x=x_{2}$, la pression dans le liquide de la fissure est : $P=P_{0}+\rho \gamma\left(x_{4}-x_{2}\right)$ et en même temps d'après la loi de Laplace :

$$
P=P_{0}+\frac{\sigma}{r_{4}}
$$

d'où

$$
\frac{\sigma}{r_{4}}=\rho \gamma\left(x_{4}-x_{2}\right) .
$$

Si le ménisque occupe partout la position d'équilibre, $r_{4}$ est une fonction décroissante de $x$, et la quantité de liquide dans la fissure diminue quand $x$ augmente. Pour prouver que cet équilibre n'est pas stable, il suffit de trouver une configuration hors d'équilibre pour laquelle l'énergie du système soit inférieure à l'énergie à la position d'équilibre. Cette configuration est représentée dans la figure $8:$ il suffit de prendre la position du ménisque symétrique de la précédente par rapport au plan $x=L / 2$. Les énergies de surface sont les mêmes que précédemment. Par contre, le centre de gravité de la masse de liquide s'est déplacé dans la direction de $\gamma$. Le système est donc plus stable dans cette position que dans la position d'équilibre. La position d'équilibre est donc instable.

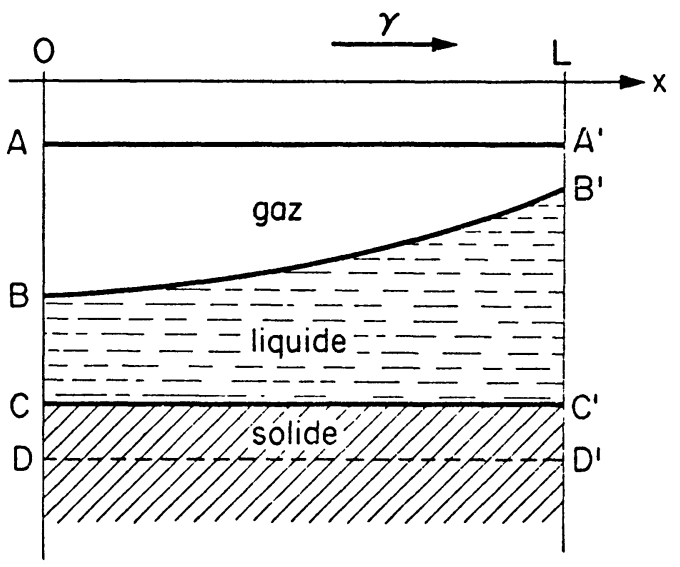

FIG. 8. - Configuration hors d'équilibre plus stable que la configuration à l'équilibre.

Du liquide qui se trouve dans une fissure n'y restera donc pas : il s'écoulera dans le sens de l'accélération ou retournera en "amont» de l'accélération se joindre à une masse de liquide située derrière un ménisque stable. Dans les deux cas, la fissure se videra. Par conséquent, chaque pore complexe restera isolé de ses voisins et la longueur caractéristique $L$ sera égale à la plus grande distance entre deux embouchures du même pore complexe, parallèlement à l'accélération.

\subsection{COMPORTEMENT D'UN LIQUIDE QUI MOUILLE BIEN} LA SURFACE EXTÉRIEURE DE LA COUCHE POREUSE. Le cas considéré ici peut se présenter si la pellicule d'oxyde qui forme normalement la surface extérieure 
du squelette poreux n'a pas pu se former, ou si elle a disparu. Le ménisque cylindrique qui surmonte une mạsse de liquide située dans une fissure est alors concave $(\theta+\beta / 2<\pi / 2)$. A l'équilibre, la pression dans le liquide au point d'abscisse $x_{4}$ est donnée par les relations :

$$
P=P_{0}-\frac{\sigma}{r_{4}} \quad \text { et } \quad P=P_{0}-\rho \gamma\left(x_{2}-x_{4}\right)
$$

d'où

$$
\frac{\sigma}{r_{4}}=\rho \gamma\left(x_{2}-x_{4}\right) \text {. }
$$

Par le même raisonnement que précédemment, on peut montrer que l'équilibre est stable et que le liquide restera dans la fissure. Le rayon de courbure du ménisque ne pourra cependant pas être inférieur à une valeur limite $r_{4 \mathrm{~min}}$ qui est liée à la forme cylindrique $\mathrm{du}$ fond de la fissure. On peut montrer facilement que cette valeur est

$$
r_{4 \min }=r_{3} \frac{\cos \beta / 2}{\cos (\theta+\beta / 2)}
$$

En amont d'un ménisque de courbure nulle, tel que le ménisque $\mathrm{M}_{2}$ considéré au paragraphe 2.2, la fissure sera donc remplie sur une longueur $L_{f}$ telle que

$$
\frac{\sigma}{r_{4 \min }}=\rho \gamma L_{f} \quad \text { soit } \quad L_{f}=\frac{\sigma \cos (\theta+\beta / 2)}{\rho \gamma r_{3} \cos \beta / 2}
$$

d'où

$$
\begin{aligned}
L_{f}=\frac{\cos (\theta+\beta / 2)}{2 \cos \theta \cos \beta / 2} \frac{(r L)_{\max }}{r_{3}} & = \\
& =\frac{(r L)_{\max }}{2 r_{3}}(1-\operatorname{tg} \theta \operatorname{tg} \beta / 2) .
\end{aligned}
$$

Ceci signifie encore que les microfissures assureront la communication continue du liquide d'un point à un autre de la surface du solide sur une longueur $L_{f}$. Ce phénomène a un effet défavorable sur la stabilité du liquide dans la couche poreuse. En effet, considérons un pore complexe caractérisé par un rayon de pore $r_{0}$ et une longueur $L_{0}$, dans lequel le liquide est stable, c'est-à-dire pour lequel $r_{0} L_{0}<(r L)_{\max }$.

Un réseau de microfissures couvrant la surface extérieure de la couche poreuse sera rempli de liquide sur une longueur $L_{f}$ telle que

$$
L_{f}=\frac{(r L)_{\max }}{2 r_{3}}(1-\operatorname{tg} \theta \operatorname{tg} \beta / 2)>\frac{r_{0} L_{0}}{2 r_{3}}(1-\operatorname{tg} \theta \operatorname{tg} \beta / 2) .
$$

Si le mouillage est bon ( $\theta$ voisin de zéro) ou si les fissures ont un profil très aigu ( $\beta / 2$ voisin de zéro), on aura

$$
L_{f}>\frac{r_{0} L_{0}}{2 r_{3}} .
$$

Comme $r_{3} \ll r_{0}$, on aura $L_{f} \gg L_{0}$.
Si les pores représentent une partie appréciable de la superficie du solide poreux, et que la distance d'un pore complexe à l'autre est du même ordre de grandeur que la dimension du pore complexe lui-même, soit $L_{0}$, la microrugosité de la surface extérieure de la couche solide aura donc pour effet de faire communiquer tous les pores entre eux. Dans ce cas, l'ensemble de la couche poreuse doit être considéré comme un pore complexe unique. La distance $L$ est alors égale à la longueur totale de la couche poreuse.

Dans le cas extrême où le mouillage du solide par le liquide est parfait $(\theta=0)$, ce qui se produit si $\sigma_{\mathrm{S}}-\sigma_{\mathrm{SL}}>\sigma_{\mathrm{L}}$ ( $\sigma$ étant l'énergie spécifique d'une surface et les indices $S$ et $L$ désignant le solide et le liquide), la stabilité du liquide dans une fissure est régie par les mêmes lois que précédemment. Cependant, la mouillabilité parfaite a une conséquence importante : un film très fin de liquide (au moins monomoléculaire) recouvre toute la surface du solide. Dans ce cas, toutes les masses de liquide situées dans les porosités ouvertes communiquent entre elles. Même en l'absence de rugosité de la surface, la distance $L$ est égale à la longueur totale de la couche poreuse.

Application à un revêtement situé sur une pièce statique. - En reprenant l'exemple considéré au paragraphe 2.2 , on voit que la rugosité de la surface extérieure de la couche poreuse et sa mouillabilité n'ont pas d'influence sur la stabilité de la phaseliquide : en effet, le liquide est stable même si la porosité est entièrement interconnectée, que l'interconnexion soit réalisée par l'intérieur de la porosité ou par la surface extérieure du revêtement.

Application à un revêtement situé sur une aube de turbine à gaz. - Supposons que le liquide mouille bien la surface extérieure du revêtement. La longueur caractéristique $L$ est alors égale à la longueur totale de l'aube, soit environ $10 \mathrm{~cm}$. En reprenant les mêmes valeurs numériques que précédemment, pour lesquelles $(r L)_{\max } \sim 10^{-5} \mathrm{~cm}^{2}$, on voit que le liquide n'est stable que dans des pores d'un rayon maximal $r_{\max } \sim 10^{-6} \mathrm{~cm}=10^{-2}$ microns. On voit donc que si le liquide mouille la surface du solide, il ne sera pas stable dans les pores que l'on peut réaliser en pratique, dont le diamètre est de l'ordre de quelques microns.

4. Influence de la rugosité de la surface intérieure du solide et de sa mouillabilité sur la stabilité de la phase liquide. - On n'a considéré ci-dessus que des situations où les pores étaient complètement remplis de liquide. La mouillabilité de la surface intérieure du solide, qui doit dans tous les cas être très bonne, n'intervient alors que par son influence sur $(r L)_{\max }$. Sa rugosité n'a pas d'influence sur la stabilité du liquide.

Le degré de mouillage et la rugosité de la surface intérieure auront cependant une influence sur la quantité de liquide qui reste dans un pore complexe qui se vide partiellement. 
En effet, considérons un pore complexe comprenant des pores élémentaires de différents rayons où le liquide n'est pas stable (Fig. 9). Au point d'abscisse $x_{0}$, le liquide n'est stable que dans les pores de rayon inférieur à $r_{0}$.

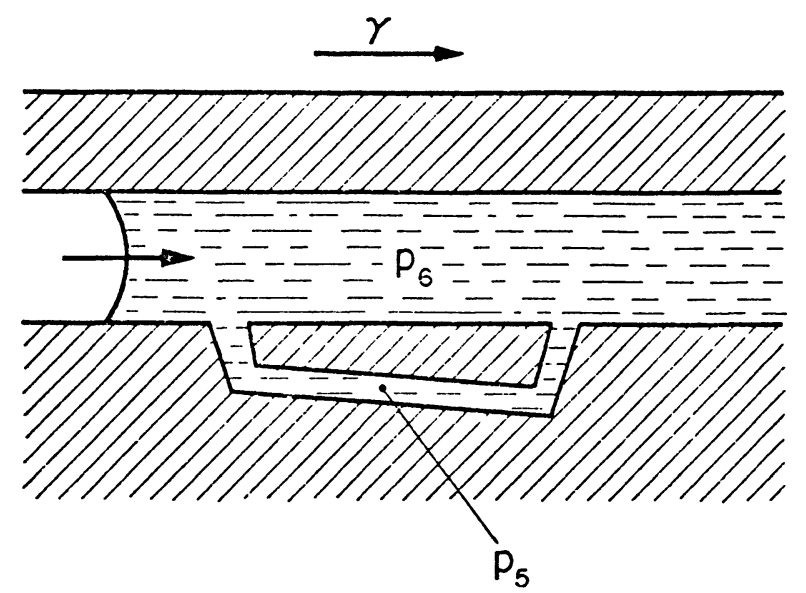

FIG. 9. - Vidage partiel d'un pore complexe.

Considérons deux pores élémentaires $\mathrm{p}_{5}$ et $\mathrm{p}_{6}$ de rayons $r_{5}$ et $r_{6}$ tels que $r_{0}<r_{5}<r_{6}$. Quand le pore complexe se videra, le pore $\mathrm{p}_{6}$ se videra en premier. Si l'angle de mouillage est nul ou si la surface des pores est rugueuse, le liquide du pore $p_{5}$ ne cessera pas de communiquer avec le liquide situé dans le reste du pore complexe. Le pore $\mathrm{p}_{5}$ se videra donc. Au contraire, si $\theta \neq 0$ et si la surface des pores est lisse, dès que le pore $p_{6}$ sera vide, le pore $p_{5}$ sera isolé et se comportera comme un pore indépendant, où le liquide sera stable. Dans ces conditions, une couche poreuse comprenant des pores de forme irrégulière ayant une large distribution de diamètres ne peut pas se vider complètement. Seuls les plus gros pores d'interconnexion se vident, les pores plus petits restent remplis de liquide.

5. Influence de la présence d'une couche d'oxyde à la surface du liquide sur la stabilité du liquide dans la couche poreuse. - On va considérer ici l'influence qu'exercent les couches d'oxyde, non plus par leur effet sur la mouillabilité, mais par leur résistance mécanique qui leur permet de barrer solidement les ouvertures de pores par où le liquide aurait tendance à s'échapper.

On suppose ici que l'épaisseur de la couche d'oxyde est du même ordre de grandeur que le rayon des pores élémentaires. Considérons une telle couche d'oxyde obturant l'embouchure d'un pore (Fig. 10).

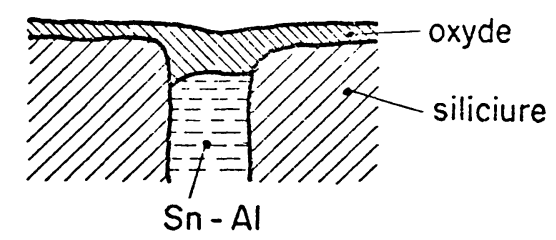

FIG. 10. - Bouchon d'oxyde à l'embouchure d'un pore.
Soit $P$ la pression du liquide dans le pore. La couche d'oxyde subit des contraintes qui sont du même ordre de grandeur que, par exemple, la contrainte de cisaillement le long des parois du pore où elle est fixée. Cette contrainte est

$$
\frac{P \cdot \pi r^{2}}{2 \pi r . r}=\frac{P}{2} .
$$

La résistance au cisaillement de l'alumine à $1200^{\circ} \mathrm{C}$ est de l'ordre de $10 \mathrm{~kg} / \mathrm{mm}^{2}$. Ceci correspond à $P=20 \mathrm{~kg} / \mathrm{mm}^{2}=2 \times 10^{9}$ dynes $/ \mathrm{cm}^{2}$. Dans le cas d'un revêtement situé sur l'aube de turbine déjà considérée plus haut, cette pression hydrostatique serait obtenue pour une longueur caractéristique

$$
L=\frac{P}{\rho \gamma}=\frac{2 \times 10^{9}}{4 \times 4 \times 10^{7}}=12,5 \mathrm{~cm} .
$$

On voit donc qu'une couche d'oxyde dont l'épaisseur est du même ordre de grandeur que le rayon des pores peut retenir le liquide dans la couche poreuse, même si la porosité est interconnectée sur une distance de l'ordre de grandeur de la longueur d'une aube de turbine.

6. Discussion des résultats et application à un alliage Sn-Al imprégnant une couche poreuse de $\mathrm{NbSi}_{2}$. Les calculs qui précèdent ont décrit le comportement $\mathrm{du}$ liquide dans diverses situations hypothétiques, chaque situation étant définie par un ensemble d'hypothèses de base arbitraire. Cela a permis de dégager et de comparer l'ordre de grandeur des forces qui déterminent la stabilité du liquide dans la couche poreuse de l'influence de diverses caractéristiques géométriques et physico-chimiques du système étudié.

On va considérer maintenant le cas réel d'un revêtement de protection du niobium contre l'oxydation constitué d'une couche poreuse de $\mathrm{NbSi}_{2}$ imprégnée d'un alliage liquide d'étain-aluminium, et rechercher parmi les diverses situations hypothétiques étudiées ci-dessus, celles qui correspondent le mieux à la réalité. Des recommandations concernant les caractéristiques à donner au revêtement seront également dégagées de l'étude précédente.

6.1 Porosité De LA COUCHE DE SILICIURE. - L'examen des coupes métallographiques des revêtements préparés indique que, dans certains cas, la porosité du siliciure est principalement interconnectée (Fig. 11a). Par contre, dans d'autres cas, la plus grande partie, sinon la totalité de la porosité profonde de la couche de siliciure est une porosité fermée (Fig. 11b). Ceci est très probablement dû à la solubilité du siliciure dans le liquide. Cette solubilité confère au siliciure une mobilité qui permet au système liquide-solide d'atteindre la configuration la plus stable, c'est-à-dire où la superficie de l'interface est la plus petite. En 
conséquence, un pore dont la section présente un étranglement se coupe en deux. Ce phénomène peut se produire pendant les traitements de préparation de la couche ou pendant la période d'oxydation.

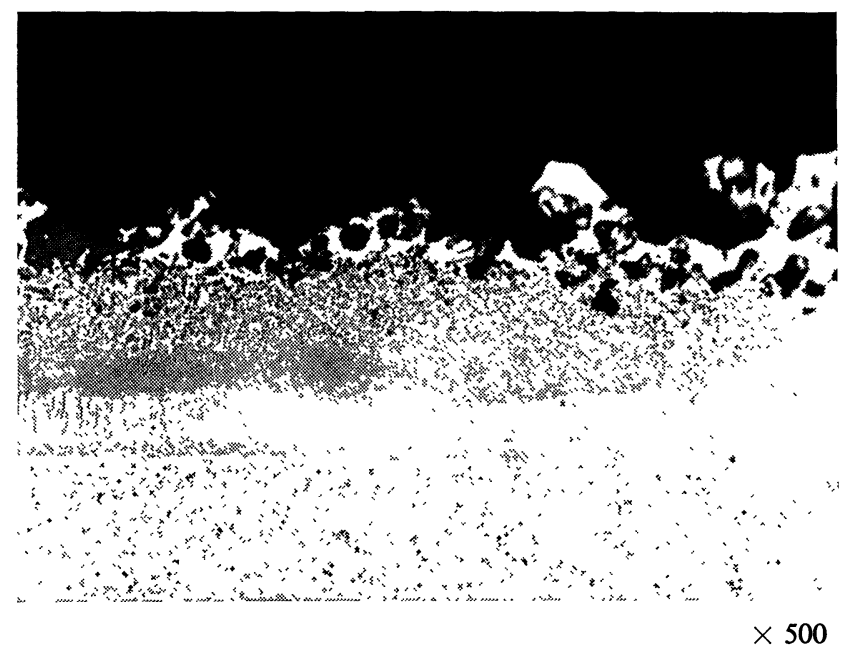

Fig. 11a. - Couche de siliciure de niobium allié imprégnée de $\mathrm{Sn}-\mathrm{Al}$ après sa fabrication (porosité principalement interconnectée).

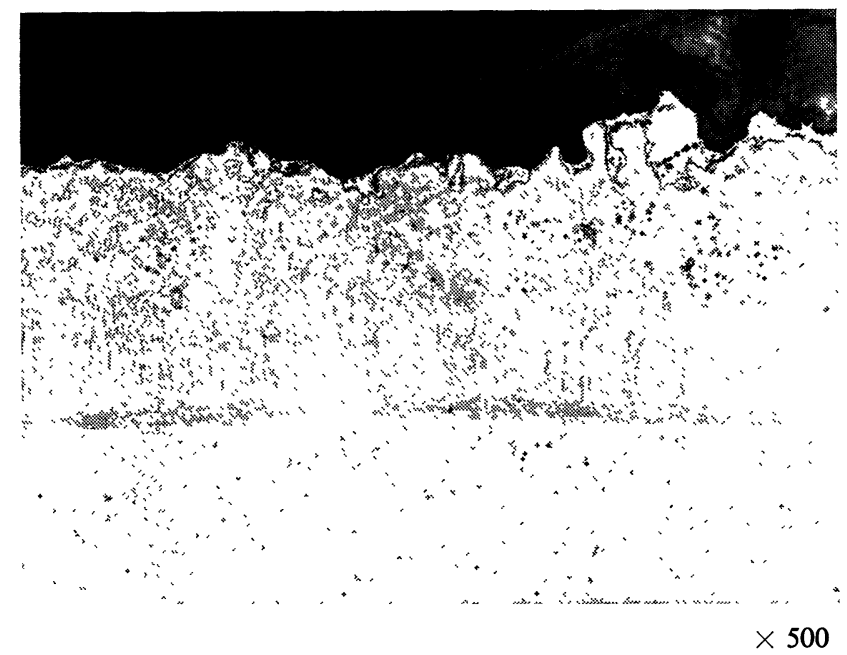

FIg. 11b. - Couche de siliciure de niobium allié imprégnée de Sn-Al après une oxydation cyclique prolongée (porosité principalement fermée).

Une porosité de type complexe a donc tendance à se séparer en plusieurs ensembles de pores complexes plus petits. D'autre part, les pores simples se scindent en plusieurs parties pour donner naissance à des pores fermés. Il est donc possible que l'incorporation, dans la préparation de la couche, d'un traitement thermique dans un domaine de température où la mobilité du siliciure est élevée, améliore la stabilité de l'alliage $\mathrm{Sn}-\mathrm{Al}$ dans la couche poreuse du siliciure.

6.2 Mouillabilité DE LA COUChe POREuSe. Pour que le liquide soit stable, il faut qu'il mouille bien la surface intérieure de la couche poreuse. Cette condition est satisfaite car l'alliage Sn-Al mouille bien le siliciure.

Dans les applications où l'accélération est forte, il faut également que le liquide ne mouille pas la surface extérieure de la couche poreuse. Cette condition est normalement satisfaite car, dès que le revêtement se trouve dans des conditions oxydantes, la surface extérieure du siliciure se recouvre d'une couche d'oxyde que l'alliage liquide mouille mal. L'alliage n'aura donc pas tendance à se répandre sur la surface extérieure du revêtement.

Il peut cependant survenir que la couche d'oxyde qui recouvre le revêtement n'ait pas pu se former ou ait disparu. Ce cas peut se rencontrer si le revêtement subit un séjour prolongé à haute température soit dans une flamme localement réductrice, soit dans un vide poussé (cas d'une application spatiale). Si le revêtement est aloìs soumis à une forte accélération, le liquide sera éjecté de la couche poreuse.

6.3 INFLUENCE DE LA COUCHE SUPERFICIELLE D'ALUMINE. - On peut voir sur les coupes métallographiques qu'après une certaine durée d'oxydation, il se forme, sur la surface extérieure du revêtement, une couche d'alumine dont l'épaisseur atteint plusieurs microns. Cette couche peut offrir une très grande résistance à la sortie du liquide dans les cas où les forces capillaires ne sont pas suffisantes pour le retenir dans la porosité.

La bonne liaison entre l'alumine et la couche de siliciure que des considérations thermodynamiques permettent de prévoir est évidemment nécessaire pour que la couche d'alumine remplisse ce rôle de barrière pour le liquide.

On peut donc prévoir qu'une étape de la fabrication du revêtement sera une oxydation contrôlée en l'absence d'accélération forte, de manière à former cette couche d'alumine épaisse et solide.

7. Conclusions et résumé. - La stabilité mécanique d'un alliage métallique liquide imprégnant une couche superficielle solide poreuse soumise à une accélération dépend :

- de la forme de la porosité, qui doit être fine et peu interconnectée,

- de la mouillabilité du solide par le liquide, qui doit être bonne à l'intérieur de la couche poreuse et mauvaise à sa surface extérieure,

- de la présence de couches superficielles d'oxyde, qui ferment les embouchures des pores.

Dans le cas d'un revêtement placé sur une aube mobile de turbine à gaz, une por osité fine et très peu interconnectée est nécessaire. Dans le cas d'un revêtement situé sur une pièce statique, une porosité fine retiendra efficacement le liquide, quel que soit son degré d'interconnexion. 
Notations utilisées :

$\rho\left(\mathrm{g} / \mathrm{cm}^{3}\right)$ $\sigma$ (dynes $/ \mathrm{cm})$ $\theta$ (degré)

$\gamma\left(\mathrm{cm} / \mathrm{s}^{2}\right)$

$R_{t}(\mathrm{~cm})$

$\omega(\mathrm{rd} / \mathrm{s})$

$\mathrm{Ox}, \mathrm{Oy}, \mathrm{Oz}$ solide ; masse spécifique du liquide;

tension superficielle du liquide ;

angle de contact du liquide avec le accélération : rayon de la turbine;

vitesse de rotation de la turbine; système d'axes tel que le plan $x O y$ est la surface du substrat solide. $O x$ est parallèle à l'accélération ; $r(\mathrm{~cm}) \quad$ rayon d'un pore cylindrique ou rayon de courbure d'un ménisque cylindrique ;

$R(\mathrm{~cm}) \quad$ rayon de courbure d'un ménisque sphérique ;

$L$ (cm) distance maximale entre deux embouchures d'un pore complexe, parallèlement à l'accélération ;

$P$ (dynes $\left./ \mathrm{cm}^{2}\right)$ pression;

$P_{0}\left(\right.$ dynes $\left./ \mathrm{cm}^{2}\right)$ pression de l'atmosphère ambiante.

Bibliographie

[1] Stecher (P.), Lux (B.), Funk (R.), Protection contre l'oxydation d'alliages de niobium, Entropie, 1968, 39.

[2] Lux (B.), Stecher (P.), Perlhefter (N.), Protection contre l'oxydation du niobium au moyen d'une couche de $\mathrm{NbSi}_{2}$ imprégnée de Sn-Al, Annales des Mines, 1966, 802.

[3] Stecher (P.), LuX (B.), Protection du niobium contre l'oxydation, Publication à paraître simultanément avec le présent texte $J$. Physique, 1970, 5, 573. 